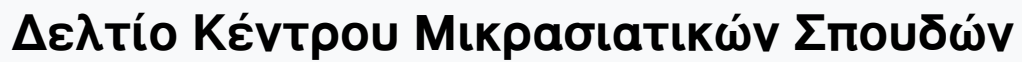

Tóp. 8 (1990)

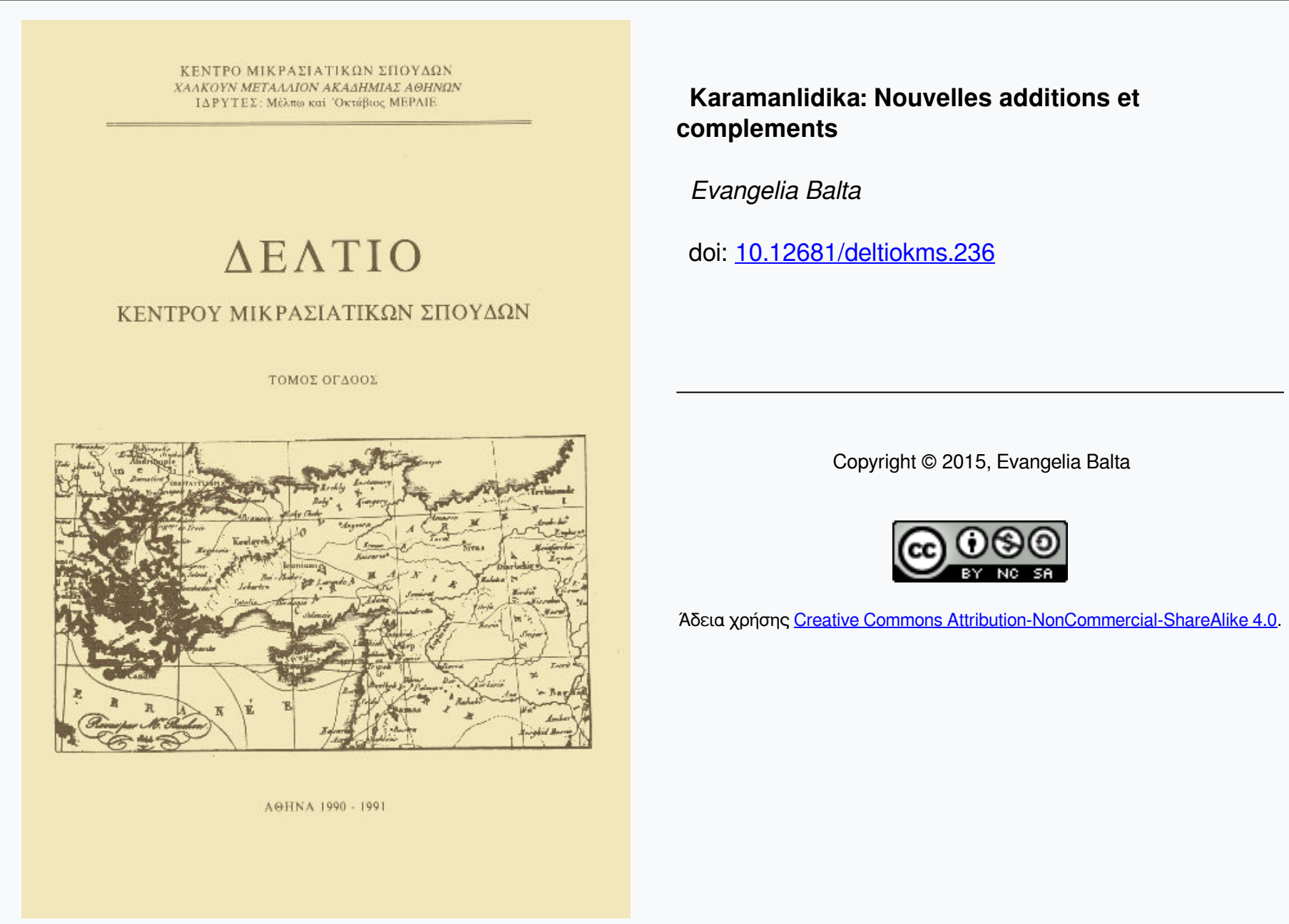

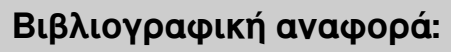

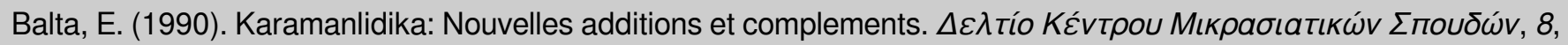
143-169. https://doi.org/10.12681/deltiokms.236 


\section{KARAMANLIDIKA NOUVELLES ADDITIONS ET COMPLÉMENTS}

Dans la préface de l'édition des Additions, j'écrivais que le nombre de 163 articles bibliographiques était «tout à fait provisoire, en raison d'une part des importantes lacunes bibliographiques qui continuent d'exister pour cette période et d'autre part du sort de ces livres, qui a suivi le destin de leur public (émigration et installation en Grèce, apprentissage de la langue grecque)». Les 17 nouvelles additions qui sont présentées ici et qui viennent s'ajouter aux 634 articles bibliographiques précédents ${ }^{1}$ témoignent de l'ampleur de la matière bibliographique qui continue à nous échapper.

Le type des nouveaux imprimés jusqu'ici non répertoriés qui sont décrits ci-dessous confirme encore une fois le sentiment que le grand livre tridimentionnel ne "se perd" pas si facilement, contrairement à la mince brochure et à la feuille d'informations d'actualité ${ }^{2}$. En dehors de ces nouveaux titres, ce qui continue à nous échapper, ce sont ces éditions en tous points similaires entre elles qui, réalisées par le même atelier typographique au cours de la même année que cette dernière, se présentent avec des éléments bibliologiques indentiques. Ce qui les en différencie peut habituellement être repéré dans la disposition des titres ${ }^{3}$ ou dans de légères modifications de la mise en page, des orne-

1. Dans les trois volumes Karamanlidika. Bibliographie analytique des ouvrages en langue turque imprimés en caractères grecs, Athènes I (1958), II (1966), III (1974), S. Salaville et E. Dalleggio ont répertorié 333 titres, en prenant pour limite extrême l'année 1900. Les 301 titres restants proviennent de E. Balta. Parmi ceux-ci, 163 constituent des additions à la bibliographie de S. Salaville et E. Dalleggio (E. Balta, Karamanlidika. Additions (1584-1900), Athènes, 1987) pour la période s'étendant jusqu'à 1900, et 138 sont des relevés concernant la production de livres du vingtième siècle (E. Balta, Karamanlidika, XXe siècle, Athènes, 1987).

2. Deux de ces feuilles sont décrites ici. Au total, dix ont été répertoriées. Cf. S. Salaville - E. Dalleggio, $\mathrm{n}^{\mathrm{os}} 29,125,251$, E. Balta, Additions, $\mathrm{n}^{\text {os }} 10,12,50,114$ et E. Balta, XXe siècle, $\mathrm{n}^{\text {os }} 92$, $93,119$.

3. Voir le cas de l'Évangile selon $S^{t}$ Luc (1905), $\mathrm{n}^{\circ} 15$. 
ments typographiques. Enfin, les rééditions étant rarement numérotées ${ }^{4}$, il est difficile de compléter telle série précise. Ainsi, les chiffres bibliographiques ne rendent pas compte des réalités de la production des livres ${ }^{5}$. Une autre remarque, qui présente de l'intérêt et qui montre de surcroît qu'il est encore trop tôt pour évaluer la production de livres karamanliens, est que 7 des 17 additions décrites sont des éditions «missionnaires». Leur nombre, aussi fortuit qu'il soit, témoigne des lacunes qui existent dans la connaissance que nous avons de l'activité des diverses Sociétés en Asie Mineure aux XIXe et XXe siècles dans le domaine de la propagande et de l'édition 6 .

On trouvera ici publiés 15 nouveaux articles bibliographiques du XIXe siècle ${ }^{7}$ et 2 du XXe. Le pourcentage le plus élevé concerne les lacunes des trois tomes de S. Salaville et E. Dalleggio et de leur complément (Additions 15841900 ), établi par E. Balta. Parmi les unités bibliographiques communiquées ici, 17 correspondent à des additions et 2 à des compléments ${ }^{8}$, c'est-à-dire des descriptions d'imprimés que nous avons eus entre les mains et qui avaient été incorporés à des publications bibliographiques antérieures à partir d'indications bibliologiques indirectes. Ces cas sont signalés par un astérisque.

En dépit de l'ampleur du travail bibliographique préalable qui a été réalisé, de nombreuses recherches complémentaires sont encore nécessaires pour parvenir, si nous devons jamais y parvenir, à un relevé de la totalité de la production des livres karamanliens. Pour combler les lacunes, il est bien entendu nécessaire de mener des recherches systématiques dans d'autres bibliothèques, de façon à repérer de nouveaux titres et éditions qui nous échappent. Les travaux de ce type n'ont pas de fin, on le sait. De même qu'il est évident qu'ils ne peuvent être l'affaire d'une seule personne. C'est avec la prière que le Bulletin du Centre d'Études d'Asie Mineure ${ }^{9}$ devienne la tribune de publication

4. Comme dans le cas de Les Actes et les Epîtres des Saints Apôtres (1804), $\mathrm{n}^{\circ} 1$.

5. En ce qui concerne la difficulté de repérer des éditions semblables en tous points,

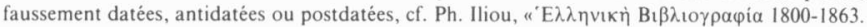

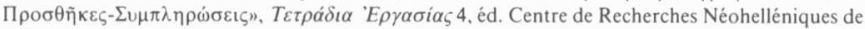
la Fondation de la Recherche Scientifique, Athènes, 1983, p. $\sigma \tau^{\prime}-\zeta^{\prime}$.

6. Sur une première tentative d'étude de la composition de la bibliographie et de la production

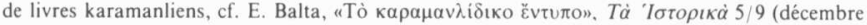
1988), p. 213-228. Dans cet article, je me référais en particulier également aux éditions "missionnaires" correspondantes: Cf. op.cit., p. 216-219, et spécialement Tableau 2. Un résumé de cet article a été publié en turc: "Karamanlıca Basılı Eserleri", Tarih ve Toplum 11/62 (1989); p. 121-123.

7. Trois articles bibliographiques sont sans date d’édition, mais selon toute vraisemblance ont été édités pendant le XIXe siècle.

8. Il s'agit des $n^{\circ s} 2$ et 16.

9. Puisque le Centre d'Études d'Asie Mineure a publié 4 des 5 tomes de la bibliographie karamanlienne. 
des éditions non recencées et non signalées à ce jour qu'est écrite cette note. Deux faits nous encouragent à formuler cette proposition:

a) Les dernières années, on remarque au niveau international un intérêt pour la littérature karamanlienne et, en général, pour la littérature en langue turque imprimée en caractères étrangers. Le nombre des chercheurs se multiplie. Cet intérêt permettra certainement de découvrir des titres et des éditions inconnus et en même temps d'eclairer le phénomène de la turcophonie. Ce début de dialogue montre qu'on peut sérieusement espérer dépasser les positions idéologiques du passé.

b) En suivant les catalogues des ventes aux enchères en Grèce et en Turquie, j'ai constaté qu'un marché financier s'était formé autour de l'imprimé karamanlien, qui, en raison de sa rareté, constitue un objet de collection à la mode dans les bibliothèques des collectionneurs. Ceci a pour effet de limiter le danger de destruction de l'imprimé karamanlien en tant que non utilisable. Toutefois les probabilités de repérer des imprimés non recensés dans ces bibliothèques demeurent faibles. Il serait souhaitable que les particuliers qui les collectionnent fassent part aux collaborateurs du Centre d'Études de l'Asie Mineure de l'existence ou de l'acquisition d'éditions non recencées ou non signalées ${ }^{10}$. L'étude du profil de cette littérature et les recherches sur la culture de son public de lecteurs, mais aussi, de façon plus générale, sur la culture de l'Hellénisme d'Asie Mineure, rendent impérieuse la nécessité de combler les lacunes quant à la production des livres karamanliens.

La récolte présentée ici est le résultat de recherches effectuées dans les bibliothèques et les archives suivantes: Bibliothèque Nationale «Cyrille et Méthode» (Sofia), Gennadius Library (Athènes) à laquelle la famille Dalleggio a fait donation du reste des documents de E. Dalleggio, Archives Générales de l'État, fonds G. Vlahoyannis (Athènes), Bibliothèque de Başvekâlet Arşivi (Istanbul). Aussi j'ai consulté les livres recensés de la bibliothèque "Coraïs» de Chio"l et des bibliothèques de Monastères Dionysiou, Xéropotamou, Vatopédi, Koutloumous de Mont-Athos ${ }^{12}$. Nous avons également fait des recherches dans les inventaires des bibliothèques publiés à partir de 1987 .

En finissant cette note, je voudrais adresser mes remerciements les plus vifs à tous ceux qui ont contribué au repérage des articles ci-dessous ou aidé à la solution des problèmes que posait leur description.

10. Le père Mélétios Sakoulidès, à qui je renouvelle ici mes vifs remerciements, en est un brillant exemple. Nous attendons avec interêt la publication des règlements des associations non répertoriées à ce jour, qui se trouvent dans sa collection.

11. Je remercie vivement $\mathrm{Ph}$. Iliou et $\mathrm{P}$. Polémi qui ont bien voulu mettre à ma disposition les photocopies des livres recensés.

12. Je remercie mon collègue Triantaphyllos Sklavénitis qui a mis à ma disposition les microfilms. 


\author{
$\mathrm{AZHZ}$ \\ AПO $T O \Lambda O \Sigma \Lambda A P H N$ \\ AME $\Lambda E P H$ \\ BE MEXTOYП $\triangle Е P H$.

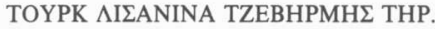

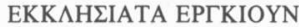 \\ OKOYNOYP. \\ BENETIKTE. 1804.
}

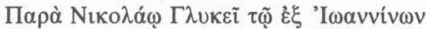

Les Actes et Les Epîtres des Saints Apôtres, mis en langue turque. Se lisent à l'église tous les jours. Venise. 1804. [En grec:] Chez Nicolas Glykys de Janina.

In-8o $(210 \times 112 \mathrm{~mm})$, de 332 pages.

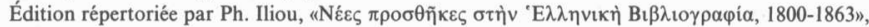

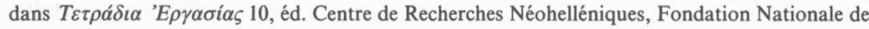
la Recherche Scientifique, Athènes 1988, p. 429, nº 715 (A 5216).

Une autre édition de cet ouvrage, connue jusqu'aujourd'hui a été parue en 1811; voir S. Salaville E. Dalleggio, $\mathrm{I}, \mathrm{n}^{\circ} 45$.

Bibl. du Musée Byzantin, Athènes.

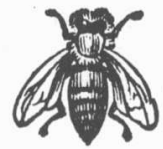




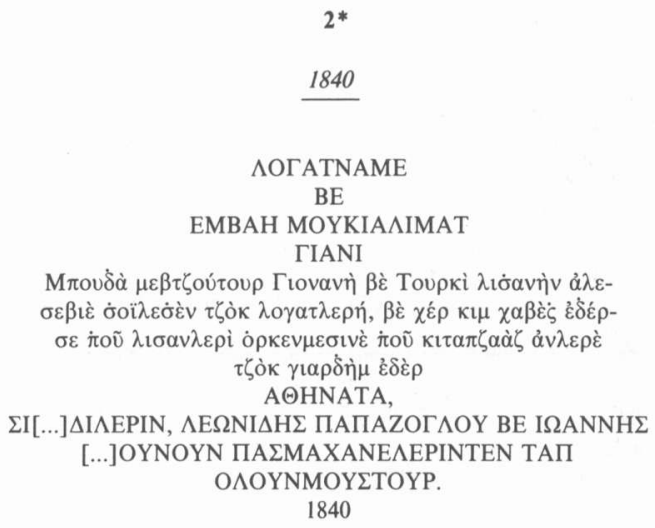

Lexique et dialogues divers. Ici sont compris lexiques en langue grecque et turque et quiconque désire apprendre les langues peut profiter largement dans ce petit livre. A Athèries, imprimé à l'imprimerie de Léonidis Papazoglou et Jean [...], 1840.

In- $8^{\circ}(200 \times 140 \mathrm{~mm})$, de 20 plus 56 pages, plus 2 feuilles au début.

F. $1^{\text {r: }}$ Titre

\section{$\Sigma Y \wedge \Lambda$ ГH \\ $\triangle I A \Phi O P \Omega N \triangle I A \Lambda O \Gamma \Omega N$ \\ ГIANI \\ ENBAГI MOYKIAAIMAT \\ METZMOYASH.}

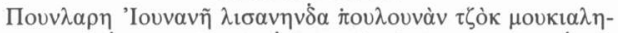

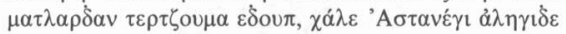

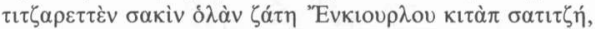

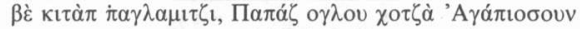

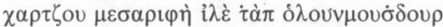

$\mathrm{A} \Theta \mathrm{HNA} \triangle \mathrm{A}$,

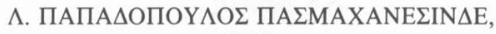
1840.

[En grec et en turc: Recueil de dialogues divers,] traduits de plusieurs livres grecs de conversation par Papazoglou, d'Ankara, actuellement commerçant libraire et relieur domicilié à Constantinople. Imprimé aux fraix d'Agapios Papazoglou, maître d'école. Athènes, à l'imprimerie de L. Papadopoulos, 1840.

Avec ce titre l'ouvrage a été répertoriée par S. Salaville - E. Dalleggio, I, $\mathrm{n}^{\circ} 102$.

F.1 $1^{\mathrm{v}}$ : blanc. 


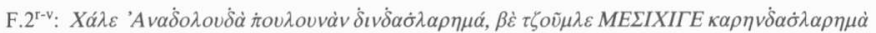
¿EAAM: Avant-propos signé par P. Sophronios.

Pages 1-20: Recueil de quatre dialogues en turc et en grec.

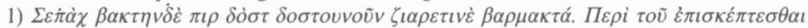
$\varphi i \lambda o \zeta \tau \iota \zeta \tau \dot{\nu} v \varphi i \lambda o v \tau o v \tau \tilde{\omega} \pi \rho \omega i$.

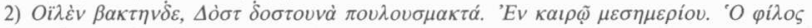

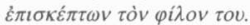

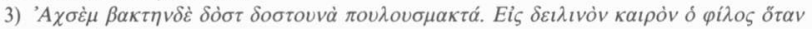

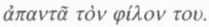

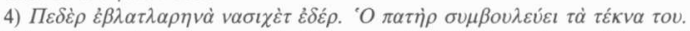

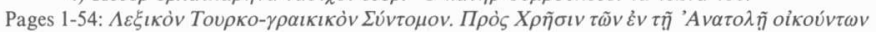
$\chi \rho \iota \sigma \tau \imath \alpha v \tilde{v} v$ : Petit lexique turco-grec à l'usage des chrétiens orthodoxes habitants de l'Asie Mineure.

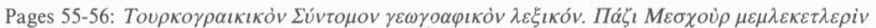

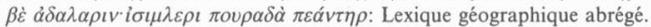

Sur la couverture de livre texte titulé XAПEPNAME dedié à la publicité de livres de Papazoglou.

Bibl. de C. Th. Dimaras, Athènes.

Bibl. de père Mélétios Sakoulidès, Istanbul.

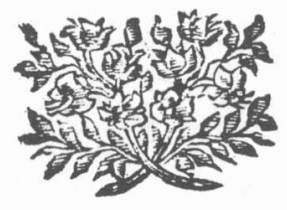


1843

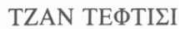 \\ ГIANI

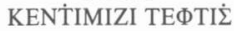 \\ ETMEK OYZEPE \\ İIP NAsIXAT

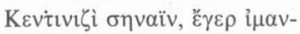

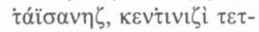

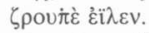 \\ B' Kop. $\imath \gamma^{\prime} .5$ \\ IZMIPTE

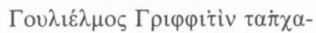

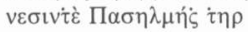 \\ 1843.
}

Examen de l'âme. C'est-à-dire exhortation à nous examiner nous-mêmes. "Examinezvous vous-mêmes, pour voir si vous êtes dans la foi, éprouvez-vous vous-mêmes”. (II Corith. 13,5). Imprimé à Smyrne, à l'imprimerie de William Griffith, 1843.

In $-8^{\circ}(133 \times 93 \mathrm{~mm})$, d'une feuille au début et 42 pages.

Signature: $2-6$,

F. $1^{\text {v: }}$ Gravure représentant un ange qui protège quelqu'un qui dort. Au-dessous la légende: «Réveille-toi, toi qui dors, et te relève d'entre les morts, et Christ t'éclairera (Éph. 5,14)».

Page 1: Titre.

Page 2: blanche.

Pages 3-42: Texte.

Bibl. Nationale "Cyrille et Méthode», Sofia, O.I.202. 


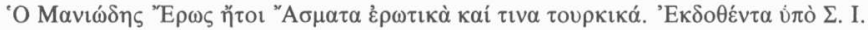

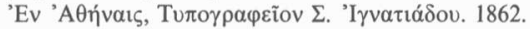

L'Éros maniaque, c'est-à-dire des chansons amoureuses et quelques turques, publiées par S. I. Athènes, imprimerie de S. Ignatiadès. 1862.

In- $16^{\circ}$, de 32 pages.

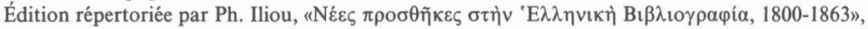

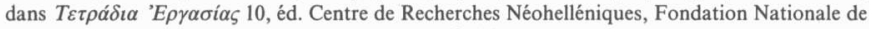
la Recherche Scientifique, Athènes 1988, p. 414, nº 638 (A 5144).

Bibl. de Manos Charitatos (seulement la couverture et les pages 31-32).

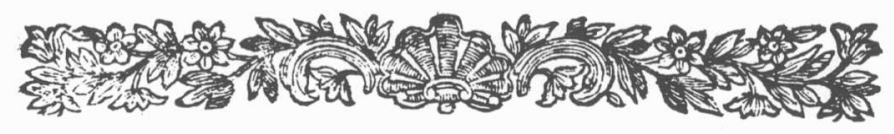




\section{$\triangle \mathrm{IA} \Lambda \mathrm{O} \mathrm{OI}$ \\ E $\Lambda$ HNO-TOYPKO-ГА $\Lambda$ О-АГГ $\Lambda$-ITA $\Lambda$ IKOI.

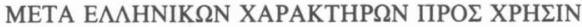

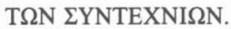

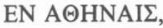

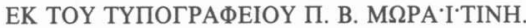
1864.

[En grec:] Dialogues gréco-turco-franco-anglo-italiens. Imprimés en caractères grecs à l'usage des corporations. A Athènes, à l'imprimerie de $P$. V. Moraitinès. 1864.

In- $16^{\circ}(120 \times 160 \mathrm{~mm})$, de 32 pages.

Pour les autres éditions de cet ouvrage voir ici les $\mathrm{n}^{0 \mathrm{~s}} 6,7,17,18,19$.

Page 1: Titre.

Page 2: blanche.

Page 3-30: Dialogues; les pages sont divisées en 5 colonnes.

Page 31: blanche. .

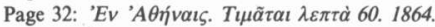

Bibl. du Centre de Recherches Néohelléniques (Fondation Nationale de la Recherche Scientifique), Athènes: 8620.

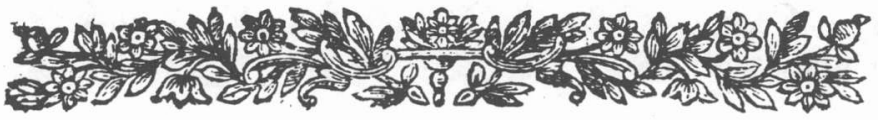




\author{
$\triangle \mathrm{IA \Lambda ОГОI}$ \\ E $\Lambda \Lambda$ HNO-TOYPKO-ГA $\Lambda$ O-АГГ $\Lambda$ O-ITA $\Lambda$ IKOI

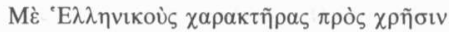

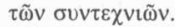

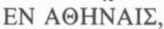

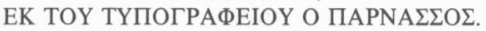

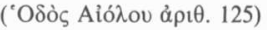 \\ 1874.
}

[En grec:] Dialogues gréco-turco-franco-anglo-italiens. Imprimés en caractères grecs à l'usage de corporations, A Athènes, à l'imprimerie "O Parnassos», 125, rue Éole. 1874.

In- $8^{\circ}$, de 32 pages.

Édition communiquée par Popi Polémi.

Pour les autres éditions connues de cet ouvrage voir ici les $\mathrm{n}^{\text {os }} 5,7,17,18,19$.

Bibl. M. Charitatos, Athènes.

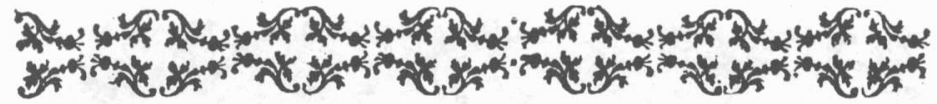




\author{
$\triangle \mathrm{IA} \Lambda \mathrm{O} \textrm{I}$ \\ E $\Lambda$ HNO-TOYPKO-ГАА О-АГГАО-ITAАIKOI.

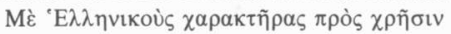

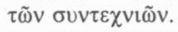 \\ EN KA $\Lambda$ AMAI $\Sigma$

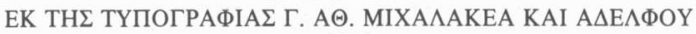 \\ 1876
}

[En grec:] Dialogues gréco-turco-franco-anglo-italiens. Imprimés en caractères grecs à l'usage des corporations. A Calames, à l'imprimerie de G. Ath. Michalakeas et frère. 1876 .

In- $16^{\circ}(110 \times 150 \mathrm{~mm})$, de 32 pages plus 2 feuilles.

Pour les autres éditions connues de cet ouvrage voir ici les $\mathrm{n}^{\text {os }} 5,6,17,18,19$.

Page 1: Titre.

Page 2: blanche.

Pages 3-31: Texte.

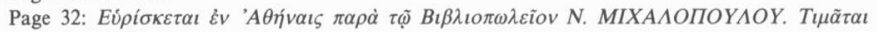
$\Lambda \varepsilon \pi \tau \dot{\alpha} 50$.

F.1: blanc.

Gennadius Library, Athènes: SL 662.31

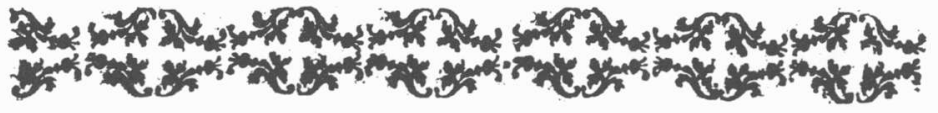


O $\Sigma \mathrm{KAN} \Delta \mathrm{A} \Lambda \Omega \Delta \mathrm{H} \Sigma \mathrm{EP} \Omega \Sigma$

HTOI

A MMATIA EPSTIKA

KAI TINA TOYPKIKA.

EN ПATPAI $\Sigma$

ТYПОГРАФЕION KAI ВІВАIOП $\Lambda$ EION «O KA $\triangle M O \Sigma »$

B. इЕКОПОY $\triangle O Y$

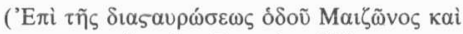

$\pi \lambda \alpha \tau \varepsilon i \alpha_{\varsigma} \Gamma \varepsilon \omega \rho \gamma$ íou $\mathrm{A}^{\prime}$.)

1882

L'Éros scandaleux, c'est-à-dire chansons amoureuses et quelques turques. A Patras, à l'imprimerie et librairie "Kadmos» de V. Sekopoulos. Au carrefour de la rue Maizon et de la place du roi Georges Ier.

In- $16^{\circ}(140 \times 110)$, de 32 pages.

Page 1: Titre.

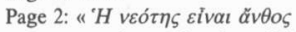

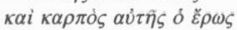

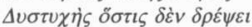

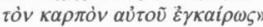

Pages 3-28: Chansons grecques.

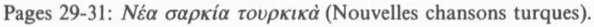

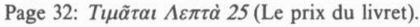

Archives Générales de l'État (Fonds de G. Vlahoyannis), Athènes.

Bibl. C. Th. Dimaras, Athènes.

Bibl. L.-M. Costakiotis, Athènes.

Gennadius Library, Athènes: MGL 932.2. 


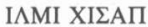

KH乏MH ZIXNI

[En caractères arabes:] Maarif-i umumiye nezaret-i celilesinin 22 Muharrem 304 ve 8 Teşrin evvel 302 tarihli ve 685 numerolu ruhsatnamesiyle ve mesarif-i amerikan misyoner şirketi tarafından tesviye olunarak tab olunmuştur

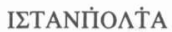

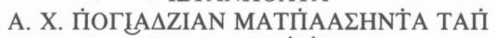

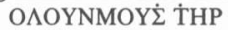 \\ 1887}

L'arithmétique. La partie de l'arithmétique mentale. Imprimé avec l'autorisation du Ministère de l'Instruction Publique $n^{\circ} 685$ du 22 Muharrem [1]304 et du 8 Teşrin evvel [1]302. Achevé d'imprimer aux frais de la Société Missionnaire américaine. Istanbul, imprimerie de A. Ch. Boyadjian, 1887.

In- $16^{\circ}(175 \times 110 \mathrm{~mm})$ de 144 pages, plus 2 feuilles blanches au début et 2 à la fin.

Édition de la Société Missionnaire américaine, qui l'année 1886 a publié un autre livre d'Arithmé-

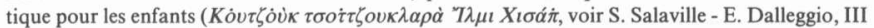
$\mathrm{n}^{\circ}$ 237).

Signature: $2-9$.

Page 1: Titre.

Page 2: blanche.

Pages 3-4: 'I $\tilde{\chi} \tau \dot{\alpha} \rho$ (Avertissement).

Pages 5-144: L'arithmétique en 42 leçons.

Bibl. du père Mélétios Sakoulidès, Istanbul. 
TAАIП BE BAIZ

TIAXOT

XE $\triangle A \Sigma$ TEPTIПI

E $\Sigma E P I$

M. ПOTZAM $\Sigma$ TENNH $\Sigma$

[En caractères arabes:] Maarif-i umumiye nezaret-i celilesinin 3 Zilkade 306 ve 19 Haziran 305 tarihli ve 331 numerolu ruhsatnamesiyle neşr olunmuștur

Mesarif-i amerikan misyoner şirketi tarafından tesviye olunarak tab olunmuştur

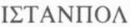

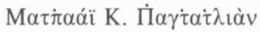 \\ ('A $\rho \alpha \mu$ iòv) \\ 1889
}

Le suppliant et le prêcher ou la modalité de salut. Ouvrage de M. Podjam Stennis. Imprimé avec l'autorisation du Ministère de I'Instruction Publique $n^{\circ} 331$, du 3 Zilkade 1306 et 19 Haziran 1305. Imprimé aux frais de la Société Missionnaire américaine. Istanbul, imprimerie du K. Baghdadlian (Aramian), 1889.

In- $12^{\circ}(165 \times 105 \mathrm{~mm})$, de 18 pages, plus 58 .

Édition de la Société Missionnaire américaine.

Pages 1-17: Commentaire sous forme questions-reponses aux verbes: $L u c X I, 13$ «Si donc vous, qui êtes mauvais, savez donner de bonnes choses à vos enfants, combien plus votre Père céleste donnera-t-il le Saint-Esprit à ceux qui le lui demandent!» et Matthieu VII, 7 "Demandez, et on vous donnera; cherchez, et vous trouverez; heurtez, et on vous ouvrira".

Page 18: blanche.

F.1: blanc.

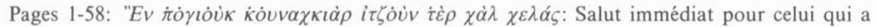
commis des péchés graves.

Bibl. du père Mélétios Sakoulidès, Istanbul.

Bibl. du Centre d'Études d'Asie Mineure, Athènes (photocopie). 
$1890-1891$

\author{
$\triangle$ OYA ХАФТАऽH \\ IN $\triangle$ ZIAI ITTIXA $\triangle$ TAPAФHN $\triangle A N$
}

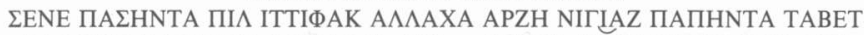

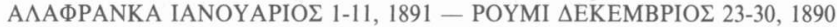

Semaine de prière, organisée par la Société Biblique. Appel à la prière à l'occasion du Nouvel An. 1-11 janvier 1891 (selon le calendrier grégorien) ou décembre 23-30 (selon le calendrier julien).

Feuille $(290 \times 200 \mathrm{~mm})$.

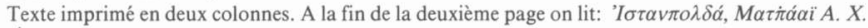

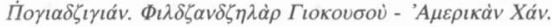

Le texte se réfère au programme de prière et aux extraits de la Bible qui seront lis et commentés chaque jour.

Bibl. du père Mélétios Sakoulidès, Istanbul.

Bibl. du Centre d'Études d'Asie Mineure, Athènes (photocopie).

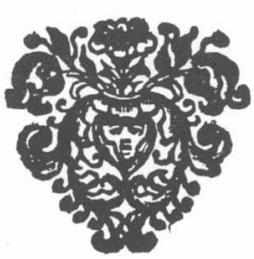




\title{
АХРЕT АZАПН
}

[En caractères arabes:] Maarif-i umumiye nezaret-i celilesinin 20 Temmuz 308 tarihli ve 215 numerolu ruhsatnamesiyle tab ve neşr olunmuştur.

Mesarif-i amerikan misyoner şirketi tarafından tesviye olumarak tab olunmuştur

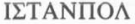

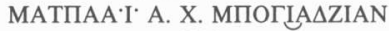 \\ 1892
}

Le jugement dernier. Imprimé avec l'autorisation du Ministère de l'Instruction Publique $n^{\circ} 215$, du 20 Temmuz [1]308. Imprimé aux frais de la Société Missionnaire américaine. Istanbul, imprimerie du A. Ch. Boyadjian, 1892.

In- $16^{\circ}(175 \times 110 \mathrm{~mm})$ de 16 pages, la dernière blanche.

Édition de la Société Missionnaire américaine.

Pages 1-15: Texte sur l'enfer.

Bibl. du père Melétios Sakoulidès, Istanbul.

Bibl. du Centre d'Études d'Asie Mineure, Athènes (photocopie). 


\title{
MÖYI்APEK KITAП்
}

[En caractères arabes:] Maarif-i umumiye nezaret-i celilesinin 12 Şevval 314 ve 5 Mart 313 tarihli ve 3 numerolu ruhsatnamesiyle tab ve neşr olunumuştur Mesarif-i amerikan misyoner şirketi tarafından tesviye olunarak tab olunmuştur

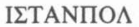 \\ MATMAAI M. ¿APIAN

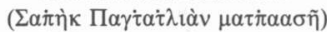

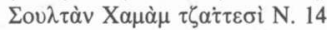 \\ 1897
}

Livre sacré. Imprimé avec l'autorisation du Ministère de l'Instruction Publique no 3, du 12 Şevval [1]314 et du 5 mart [1]313. Imprimé aux frais de la Société Missionnaire américaine. Istanbul, imprimerie du M. Saryan (l'ancienne imprimerie de Baghdadlian), $n^{\circ} 14$ avenue Sultan Hammam, 1897.

In- $8^{\circ}(135 \times 95 \mathrm{~mm})$ de 14 pages.

Éditon de la Société Missionnaire américaine.

Page 1: Titre. Vignette: balance.

Page 2: blanche.

Pages 3-13: Texte.

Page 14: blanche.

Bibl. du père Mélétios Sakoulidès, Istanbul.

Bibl. du Centre d'Études d'Asie Mineure, Athènes (photocopie). 


\section{$\mathrm{I} \cdot \mathrm{I} \cdot \Lambda \mathrm{AN}$}

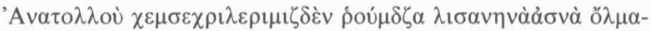

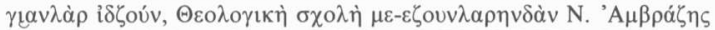

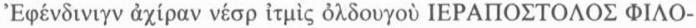

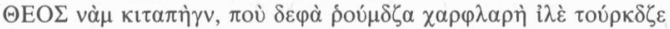

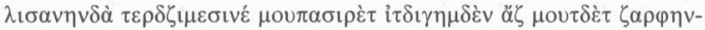

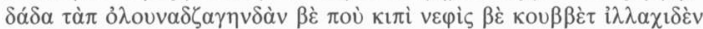

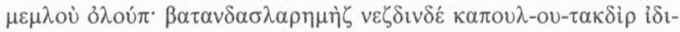

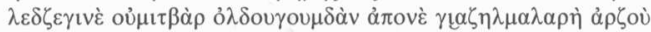
ỏhouvoúp.

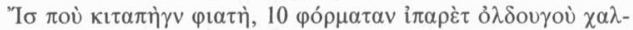

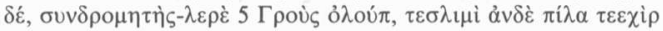

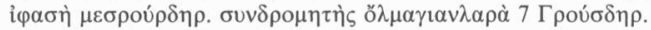

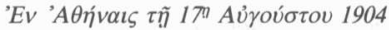

\section{I $\Omega A N N H \Sigma$ A. ПAПAAKAKIA $\Delta \mathrm{H} \Sigma$}

[Résumé:] L'annonce de la parution en karamanli de l'ouvrage Philothéos fait par Nicolas Ambrazis. Appel au peuple pour souscrire un abonnement au livre. Selon l'annonce le livre est supposé avoir 10 cahiers et son prix sera de 5 gurush (pour les souscripteurs) et de 7 gurush pour les non-souscrits. L'annonce a été imprimée à Athènes et datée en août 1904. Le chiffre du jour d'août n'est pas imprimé, mais ajouté à la main. A la fin on lit le nom de Jeanne A. Papaakakiadès.

Feuille $(280 \times 200 \mathrm{~mm})$.

L'édition grecque de Philothéos a été publiée à Athènes en 1903.

Nous connaissons que l'édition karamanlie en question a finalement paru en 1913, éditée à l'imprimerie des frères Misaelidès. (Voir E. Balta, XXe siècle, $\mathrm{n}^{\circ}$ 99).

Bibl. du Centre d'Études d'Asie Mineure, Athènes (photocopie). 
INTZIA

TIANI

EYAГГЕ $\Lambda$ ION

$\triangle O Y K A \Sigma H N$

TAXPIPI OYZPE

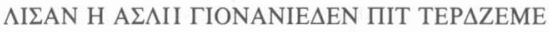

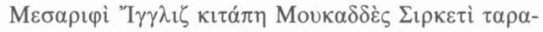

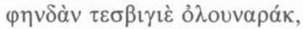

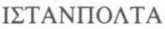

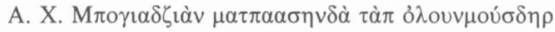
1905

L'Évangile selon Luc. Traduit de l'original grec. Imprimé aux frais de la Société Biblique anglaise. Istanbul, à l'imprimerie de A. Ch. Boyadjian, 1905.

In- $16^{\circ}(135 \times 90 \mathrm{~mm})$ de 100 pages plus 3 feuilles.

Édition de la Société Biblique. Il s'agit d'un autre tirage de l'édition de la même année faite par la Société Biblique à la même imprimerie. Le corps de l'édition présentée ici est identique à celle-ci; seulement le feuillet du titre est différent (pour la comparaison voir E. Balta, XXe siècle, $\mathrm{n}^{\circ} 23$ ).

F.1: blanc.

F.2 $2^{\text {r: blanc. }}$

F.2 $2^{v}$ : Graeco-Turkish. The Gospel According to St. Luke, 5000 copies 1905.

Page 1: Titre.

Page 2: blanche.

Pages 3-100: Texte divisé en 24 chapitres.

F.1: blanc.

Bibl. du père Mélétios Sakoulidès, Istanbul. 


\author{
OYNAIOY KIOPOГАOY \\ $\mathrm{BE}$ \\ A¿IK ГАРIП \\ XIKIAГEАEPI \\ BE \\ $\Lambda$ ETA I $\cdot$ 'Ф XАГIA $\Lambda$ \\ TAП E $\triangle I \triangle Z I \Sigma I$

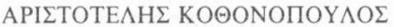 \\ $\triangle E P \Sigma A A \triangle E T \triangle E$ \\ $\triangle$ HMHTPIO $\Theta \Omega M A \cdot I \cdot \Delta H \Sigma$ \\ 1909
}

Les contes du fameux Kieuroglou et d'Achik Garip et légendes. Éditeur: Aristote Kothonopoulos. Constantinople, Dimitrios Thomaïdis, 1909.

In- $8^{\circ}(190 \times 140 \mathrm{~mm})$, de 32 pages.

Cette édition a été répertoriée dans la bibliographie karamanlienne (voir E. Balta, XXe siècle, $\mathrm{n}^{\circ}$ 49). La référence était fondée sur une mention bibliographique indirecte. Cette fois-ci, la description provient d'un examen de visu.

Page 1: Préface dans lequel l'éditeur explique qu'il publie le texte de Kieuroglou, parce qu'il est très populaire, tandis qu'il n'est pas moral. Il est très interéssant de voir exprimé pour la première fois l'avis suivant lequel le texte de Kieuroglou est particulièrement audacieux. Finalement, le texte édité est censuré et incomplet. En plus, malgré le fait que dans le titre il est .écrit que le livret comprend aussi l'édition d'Achik Garip, son texte n'est pas édité.

Page 2: blanche.

Pages 3-26: Texte de conte de Kieuroglou. Le texte n'est pas complet, comme on peut le lire dans le dernier paragraphe de la page 26 (IZAXAT).

Pages 27-31: Vers du poème Bir Sultan Abdal.

Sur la couverture du livre on lit:

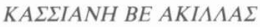

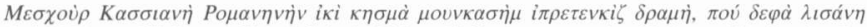

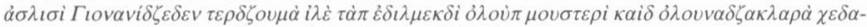

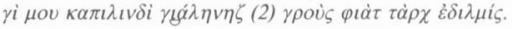

\title{
ГЕNOBEФA
}

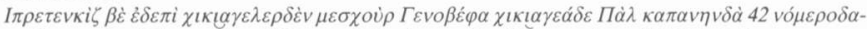

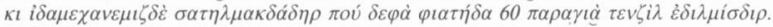

\section{NA $P E \triangle \triangle I N X O T Z A$}

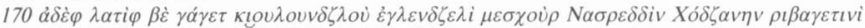

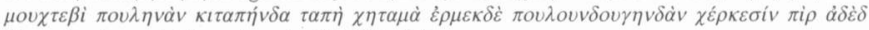
$\dot{\alpha} \lambda \mu \alpha \kappa \lambda \eta \kappa \lambda \alpha \rho \eta v \dot{\alpha}(2) \gamma \rho \sigma \dot{\nu} \varsigma \varphi \dot{\alpha} \tau \beta \dot{\alpha} \zeta \dot{\varepsilon} \delta t \lambda \mu i \sigma \delta t \rho$.

De cette liste:

L’édition de Nasreddin Hodja est parue en 1910 (voir E. Balta, XXe siècle, n 58). Pour l'édition de 
Geneviève nous pouvons dire avec certitude d'après l'information $\Pi \dot{\alpha} \lambda \kappa \alpha \pi \alpha v \eta v \delta \dot{\alpha} 42$ vó $\mu \varepsilon \rho \circ \delta \alpha \kappa \iota$ ... $\sigma \alpha \tau \eta \lambda \mu \alpha \kappa \delta \alpha ́ \delta \eta \rho$, qu'il s'agit d'une édition de l'imprimerie «Nouvelle Anatolie» de Misaélidès frères. Leur imprimerie était à Bal Kapan Han n ${ }^{\circ} 42$ (pour l'adresse voir E. Balta, op.cit, $\mathrm{n}^{\text {os }} 86$, 99). En ce qui concerne l'édition de Cassiani nous connaissons seulement une édition karamanlie jusqu’à ce jour parue en 1912 sous un title différent (voir, E. Balta, op.cit, n 81 ).

Başvekâlet Arşiv Dairesi Kütüphanesi, Istanbul.

Bibl. du Centre d'Études d'Asie Mineure, Athènes (photocopie).

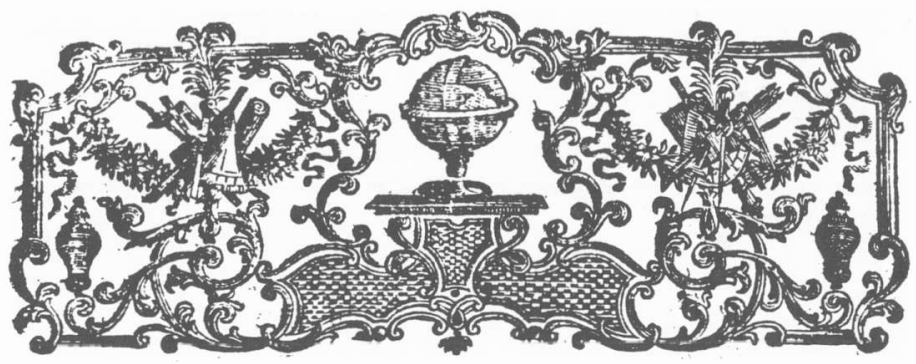




\title{
SANS DATE D’ÉDITION
}

\author{
$\triangle \mathrm{IA} \Lambda \mathrm{OГOI}$

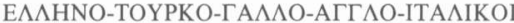

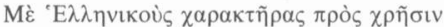 \\ $\tau \tilde{\omega} \vee \sigma u \vee \tau \varepsilon \chi \vee เ \tilde{\nu}$ \\ EK $\triangle \mathrm{I} \triangle \mathrm{OTAI}$ \\ YПO T $\Omega$ N KATA THHMAT $\Omega N$ \\ ANE $\Sigma T H ~ K \Omega N \Sigma T A N T I N I \Delta O Y$
}

[En grec:] Dialogues gréco-turco-franco-anglo-italiens. Imprimés en caractères grecs à l'usage des corporations. Publiés par la Maison d'édition d'Anestis Constantinidès.

In- $16^{\circ}$, de 32 pages.

Édition communiquée par P. Polemi.

Pour les autres éditions connues de cet ouvrage voir ici les $n^{\text {os }} 5,6,7,17,19$.

Bibl. du Centre de Recherches Néohelléniques, (Fondation Nationale de la Recherche Scientifique), Athènes: 4353 . 


\title{
$\triangle \mathrm{IA} \Lambda \mathrm{OГOI}$ \\ E $\Lambda \Lambda$ HNO-TOYPKO-ГА $\Lambda$ О-АГГ $\Lambda$ O-ITA $\Lambda$ IKOI \\ ME E $\Lambda$ HNIKOY $\Sigma$ XAPAKTHPA $\Sigma$

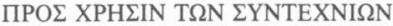

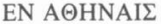

\author{
BIB $\Lambda$ IOח $\Omega \Lambda$ EION

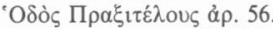 \\ ТҮПОГРАФЕІОN

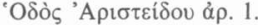 \\ ANT $\Omega$ NIOY $\Sigma$ T. ГE $\Omega$ PГIOY
}

[En grec:] Dialogues gréco-turco-franco-anglo-italiens. Imprimés en caractères grecs à l'usage des corporations. A Athènes, [Maison d'édition] d'Antoine St. Georgiou. Librairie, 56, rue de Praxitèle. Imprimerie, 1, rue d'Aristide.

In- $16^{\circ}$, de 32 pages.

Édition communiquée par P. Polemi.

Pour les autres éditions connues de cet ouvrage voir ici les ${ }^{\text {os }} 5,6,7,17,19$.

Bibl. de l'Institut Néohellénique à la Sorbonne, Paris: Br. 123.2. 


\author{
$\triangle \mathrm{IA} \Lambda$ ОГОI

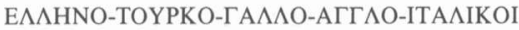

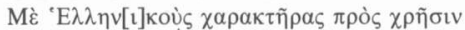

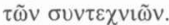

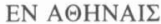 \\ ТҮПОГРАФЕION KAI ВIВ $\Lambda$ IOП $\Omega \Lambda$ EION \\ $\Delta$. A. $\Phi \mathrm{E} \Xi \mathrm{H}$ каi $\mathrm{YI} \Omega \mathrm{N}$.
}

[En grec:] Dialogues gréco-turco-franco-anglo-italiens. Imprimés en caractères grecs à l'usage des corporations. A Athènes. Imprimerie et librairie de D. A. Phexis et fils.

In- $16^{\circ}(115 \times 160)$ de 32 pages.

Pour les autres éditions connues de cet ouvrage voir ici les $\mathrm{n}^{\text {os }} 5,6,7,17,18$.

Page 1: Titre.

Page 2: blanche.

Pages 3-31: Texte.

Page 32: Gravure.

Gennadius Library, Athènes: SL 662.31. 


\section{INDEX}

Les chiffres en noir correspondent aux numeros des unités bibliographiques

\section{Achik Garip 162}

Ambrazis 160 v. Philothéos

"Anatolie Nouvelle» v. Imprimeries

Ankara 147

Aramian v. Baghdadlian

Archives Générales de l'État 145, 154

Aristide, rue 165

arithmétique 155

Asie Mineure 144, 145, 148

associations 145

Athènes

bibliothèques $146,148,150,151,152,153$, $154,156,157,158,159,160,163,164$, 166

lieu d'édition 147, 150, 151, 152, 160, 165, 168

avertissement 155

Baghdadlian K. v. Imprimeries

Bal Kapan Han 163

Balta E. 143; v. Karamanlidika. Additions (1584 -1900), Karamanlidika, XXe siècle, "Tò

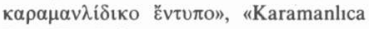
Basılı Eserleri»

Başvekâlet Arşivi v. Bibliothèques

Bible 157

Bibliothèques

Başrekâlet Arşivi 145, 163

Centre d'Études d'Asie Mineure 156, 157, $158,160,163$

Centre de Recherches Néohelléniques 151, 164

Charitatos M. 150, 152

"Coraïs" 145

Costakiotis L.-M. 154

Dimaras C. Th. 148, 154

Fondation Nationale de la Recherche Scientifique v. Centre de Recherches Néohelléniques

Gennadius 145, 153, 154, 166

Institut Néohellénique à la Sorbonne 165

Monastère Dionysiou 145

\author{
Monastère Koutloumous 145 \\ Monastère Vatopédi 145 \\ Monastère Xéropotamou 145 \\ Musée Byzantin 146 \\ Nationale "Cyrille et Méthode» 145, 149 \\ Sakoulidès Mélétios 148, 155, 156, 157, \\ 158,159
}

Bir Sultan Abdal 162

Boyadjian A. Ch. v. Imprimeries

Bulletin du Centre d'Études d'Asie Mineure 144

Calames 153

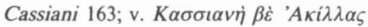

catalogues des ventes 145

Centre d'Études d'Asie Mineure 144, 145; v. Bibliothèques, Bulletin

Centre de Recherches Néohelléniques v. Bibliothèques

chansons

grecques 154

turques 154

L'Éros maniaque 150

L'Éros scandaleux 154

Charitatos M. v. Bibliothèques

Chio v. Bibliothèques

chrétiens orthodoxes 148

Christ 149

collectionneurs 145

"Coraïs" v. Bibliothèques

corporations 151, 152, 153, 165, 166

Costakiotis L.-M. v. Bibliothèques

Costantinidès A. v. Imprimeries

Costantinople 162

«Cyrille et Méthode» v. Bibliothèques

Dalleggio E. 145; v. S. Salavillę - E. Dalleggio

Dialogues 148, 151, 152, 153, 164, 166

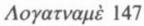

Dialogues gréco-turco-franco-anglo-italiens $151,152,153,164,165,166$

Dimaras C. Th. v. Bibliothèques

Dionysiou, monastère v. Bibliothèques 
éditeurs

Georgiou A. St. 165

I. S. 150

Kothonopoulos A. 162

Papazoglou A. 147

Société Biblique 157, 161

Société Missionnaire américaine 155, 156, 158,159

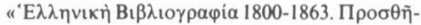

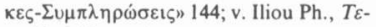

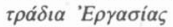

émigrations 143

enchères 145

Éole, rue 152

Évangile v. Gospel

Fondation Nationale de la Recherche Scientifique v. Centre de Recherches Néohelléniques

Fonds G. Vlahoyannis v. Archives Générales de l'État

Geneviève 163

Georges Ier, place 154; v. Patras

Glykys N. v. Imprimeries

Gospel 161

gravure 149,166

Grèce 145

Griffith W. v. Imprimeries

gurush 160

Hellénisme 145

I. S. 150; v. éditeurs, Imprimeries

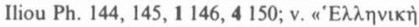

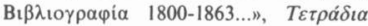

Imprimeries

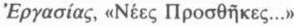

Aramian v. Baghdadlian K.

Baghdadlian K. 156

Boyadjian A. Ch. 155, 157, 158, 161

Costatninidis A. 164

Georgiou A. St. 165

Glykys N. 146

Griffith W. 149

Ignatiadès S. 150

"Kadmos» 154; v. Sekopoulos V.

Michalakeas $G$. Ath. et frère 153

Misaélidès frères 160,163

"Nouvelle Anatolie» 163; v. Misaélidès frères
"O Parnassos" 152

Papazoglou L. et Jean [...] 147

Phéxis D. A. et fils 166

Sekopoulos V. 154; v. "Kadmos"

Thomaïdis D. 162

installation 143

Istanbul

bibliothèques $148,155,156,157,158,163$

lieu d'édition 155, 156, 157, 158, 159, 161

Institut Néohellénique à la Sorbonne v. Bibliothèques

Janina v. Glykys N.

"Kadmos» v. Imprimeries

"Karamanlıca Basılı Eserleri» 144; v. Tarih ve Toplum, Balta E.

Karamanlidika, Additions (1584-1900) 143, 144; v. Balta E.

Karamanlidika. Bibliographie analytique des ouvrages en langue turque imprimés en caractères grecs 143; v. Salaville S. - Dalleggio $\mathrm{E}$.

Karamanlidika. XXe siècle 143, 14 160, 15 161, 16162,163 ; v. Balta E.

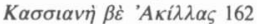

Kieuroglou 162

Kothonopoulos A. 162

Koutloumous, monastère v. Bibliothèques

langues

grecque 143

turque 145

légende 148

L'Éros manianque v. Chansons

L'Éros scandaleux v. Chansons

Les Actes et les Epîtres des Saintes Apôtres 144 lexiques

géographique 148

turco-grec 148

littérature 145

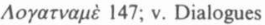

Maizon, rue 154 ; v. Patras

Matthieu 156

Michalakeas G. Ath. et frère v. Imprimeries

Ministère de l'Instruction Publique 155, 156, 158,159

Misaélidès frères 160,163 ; v. Imprimeries

missionnaires 144, 155, 156, 158, 159; v. Sociétés missionnaires, Société Missionnaire amé- 


\section{ricaine}

éditions 144

propaganda 144

Mount Athos 145; v. Monastères

Musée Byzantin v. Bibliothèques

\section{Nasreddin Hodja 162}

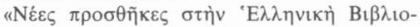

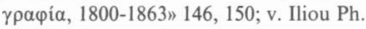

«Nouvelle Anatolie» v. Imprimeries

«O Parnassos» v. Imprimeries

Papaakakiadès J. A. 160

Papazoglou A. 147, 148

Papazoglou L. 147; v. Imprimeries

Paris 165

Patras 154

péchés 156

Phéxis D. A. et fils 166; v. Imprimeries

Philothéos 160

Polemi P. 145, 152, 164, 165

Praxitèle, rue 165

règlement 145

\section{Saint-Esprit 156}

Saint Luke v. Évangile

Sakoulidès M. 145; v. Bibliothèques
Salaville S. - Dalleggio E. 143, 144, 1 146, 2 147, 9155

Sekopoulos V. v. Imprimeries

Sklavénitis T. 145

Smyrne 149

Société Biblique 157, 161

Société Missionnaire américaine 155, 156, 158, 159

Sofia 145, 146; v. "Cyrille et Méthode»

Sophronios P. 148

Sorbonne v. Institut Néohellénique à la Sorbonne

souscripteurs 160

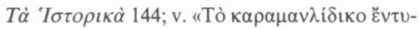
$\pi \mathrm{n})$

Tarih ve Toplum 144; v. Balta E.

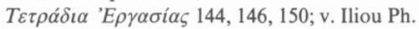
Thomaïdis D. 162

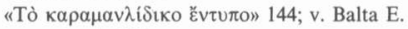
turcophonie 145

Turquie 145

Vatopédi, monastère v. Bibliothèques

Venise 146

vignette 159

Vlahoyannis G. v. Archives Générales de l'État

Xéropotamou, monastère v. Bibliothèques 